\title{
Image of the month: Diagnostic and therapeutic challenges in the management of ectopic ACTH syndrome: a perfect storm of hypercortisolism, hyperglycaemia and COVID-19
}

\author{
Author: Tejhmal Rehman ${ }^{\mathrm{A}}$
}

Cushing's syndrome is a rare endocrine disorder requiring a high degree of clinical suspicion and meticulous investigations to diagnose and manage optimally. Delayed diagnosis and suboptimal treatment are associated with increased morbidity and mortality. Uncontrolled hypercortisolism leads to multiple cardiovascular and metabolic complications. Key risk factors identified for COVID-19-related adverse clinical outcomes (such as diabetes, hypertension and obesity) are, in fact, core clinical manifestations of Cushing's syndrome. Occurrence of SARS-CoV-2 infection in someone with uncontrolled hypercortisolism could, therefore, lead to disastrous medical consequences. We report a case highlighting challenges in the diagnosis and management of aggressive Cushing's syndrome secondary to ectopic adrenocorticotropic hormone (ACTH) secretion from an, as yet unidentified, neuroendocrine tumour. Our patient also contracted SARS-CoV-2 infection during investigations, which posed additional difficulties with aggravation of cardiometabolic complications. We also identify lack of clinical evidence to address management of this unique combination of two potentially life-threatening illnesses.

KEYWORDS: ectopic ACTH syndrome, hypercortisolism, hyperglycaemia, COVID-19

DOI: $10.7861 /$ clinmed.2021-0005

\section{Introduction}

Cushing's syndrome is characterised by a constellation of clinical features and associated comorbidities caused by chronic exposure to excess glucocorticoids. While exogenous (iatrogenic) steroid use remains the common cause, endogenous hypercortisolism is rare, with an estimated incidence of $0.7-2.4$ cases per million per year. ${ }^{1}$ Broadly, hypercortisolism is categorised into adrenocorticotropic hormone (ACTH)-dependent versus independent causes with the latter primarily caused due to autonomous adrenal pathology.

Author: ${ }^{\text {A }}$ consultant endocrinologist and assistant professor of endocrinology, Shifa International Hospital, Islamabad, Pakistan
ACTH-dependent hypercortisolism due to pituitary adenoma (termed as 'Cushing's syndrome') remains the most common cause $(80 \%)$ with the rest of the cases caused by ectopic ACTH or, more rarely, corticotropin releasing hormone (CRH) secretion. ${ }^{2}$ In practice, confirming endogenous hypercortisolism and determining underlying cause is fraught with complexities and management challenges, even in the best of healthcare settings with many diagnoses delayed for months to years. This is, in part, due to non-specificity and variable manifestations of clinical features causing the patient to present to various specialties before the penny drops and correct diagnosis comes to the fore.

The case presented here came to the attention of the treating endocrinologist through a chance encounter outside of clinical settings. It led to stormy clinical course, posing complex diagnostic and therapeutic challenges in resource constrained settings amid a global pandemic, further complicated by SARS-CoV-2 infection and metabolic decompensation with eventual radical therapeutic measures leading to the path of recovery.

\section{Case presentation}

A 28-year-old man presented to the endocrine clinic with a 6-month history of rapid weight gain (approximately $20 \mathrm{~kg}$ ), fatigue, generalised muscle and joint pains, intermittent headaches and low mood. Prior to presentation, he was seen by various physicians and received diagnoses of hypertension, pre-diabetes, depression and sleep apnoea. There was no other past medical history of note. He was not taking any regular medications and denied steroid use in any form. There was no drug allergy of note. He was married, worked as shopkeeper and smoked occasionally. There was no history of illicit drug usage. He had a family history of diabetes on paternal side.

Clinically, he displayed cushingoid features with rounded face, plethora, hyperpigmentation, acne and a supraclavicular fat pad (Fig 1a left). He also had abdominal adiposity with wide (over $3 \mathrm{~cm}$ ) violaceous striae over the abdomen (Fig 1b). There was significant lower limb wasting with demonstrable proximal myopathy. Blood pressure was $150 / 90 \mathrm{mmHg}$, weight at $87 \mathrm{~kg}$ with height of $171 \mathrm{~cm}$ (body mass index (BMI) of $28 \mathrm{~kg} / \mathrm{m}^{2}$ ).

He underwent $1 \mathrm{mg}$ overnight dexamethasone test followed by 2 days of low-dose dexamethasone suppression test (LDDST), both of which failed to show suppression with cortisol levels of $979 \mathrm{nmol} / \mathrm{L}$ and 1,324 $\mathrm{nmol} / \mathrm{L}$, respectively (normal suppression 

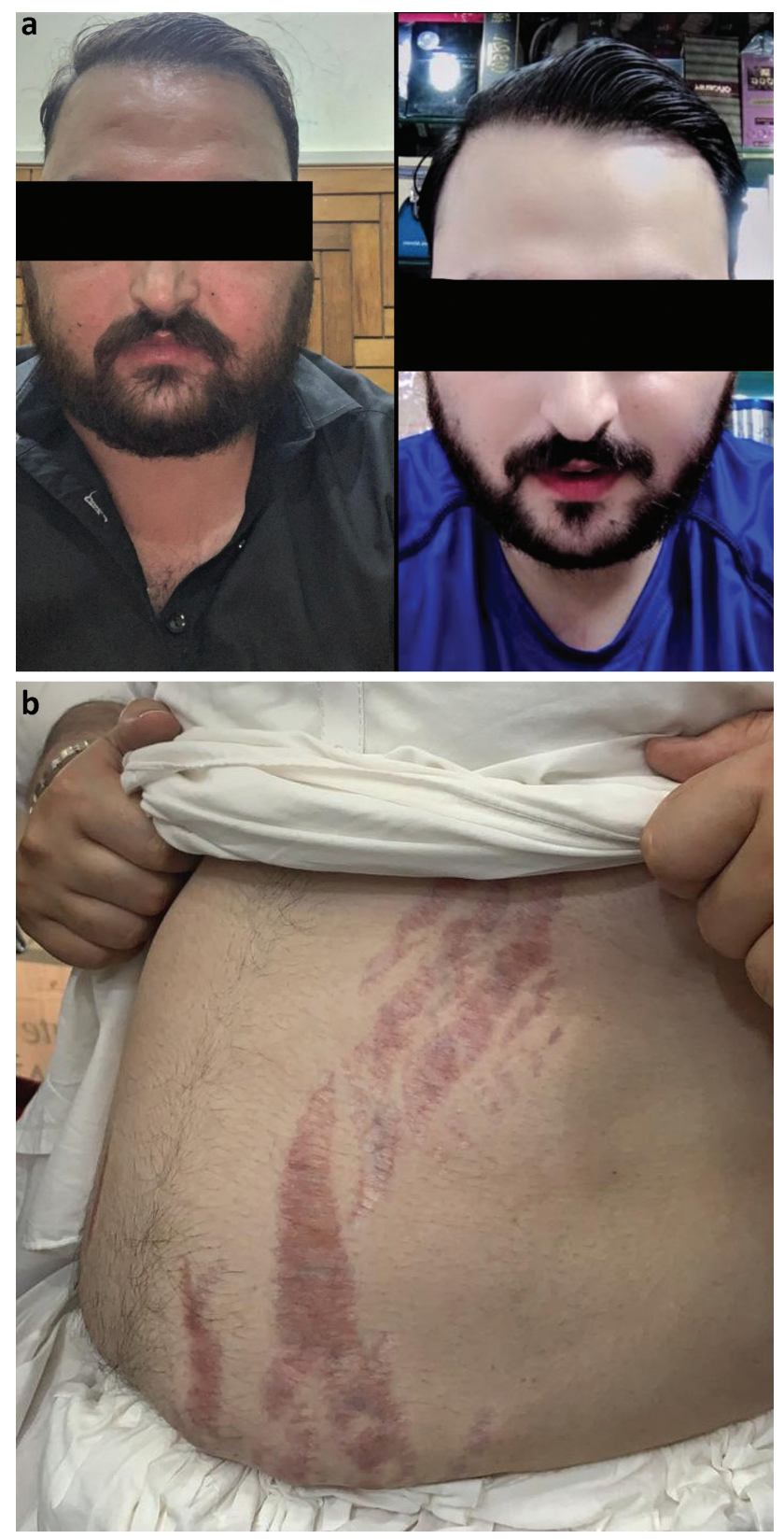

Fig 1. Photographs of presenting features. a) Left: Cushingoid features with rounded face, plethora, hyperpigmentation, acne and supraclavicular fat pad. a) Right: Before onset of symptoms for comparison. b) Abdominal adiposity with wide (over $3 \mathrm{~cm}$ ) violaceous striae over the abdomen.

$<50 \mathrm{nmol} / \mathrm{L}$ ). ACTH level was high at $81.8 \mathrm{pg} / \mathrm{mL}$ (normal range (NR) 7-69), thus confirming ACTH-dependent hypercortisolism. Other tests showed glycated haemoglobin $(\mathrm{HbA1c})$ at $7.5 \%$, alanine aminotransferase (ALT) $55 \mathrm{U} / \mathrm{L}$, hypertriglyceridemia 170 $\mathrm{mg} / \mathrm{dL}(\mathrm{NR}<150)$. Magnetic resonance imaging of the pituitary fossa did not reveal any obvious pituitary adenoma.

His presentation to the endocrine clinic coincided with the national lockdown due to global coronavirus pandemic in April and all routine hospital services were suspended at that time. He was started on ketoconazole $800 \mathrm{mg}$ daily to control hypercortisolism.
Additionally, he was started on metformin $1 \mathrm{~g}$ twice daily (BD), gliclazide modified release $30 \mathrm{mg}$ once daily (OD), atorvastatin $10 \mathrm{mg}$ OD, escitalopram $10 \mathrm{mg}$ OD, and amlodipine and valsartan combination of 5/160 mg OD. Inferior petrosal sinus vein sampling (IPSS) was planned as soon as interventional radiology services become operational.

\section{Clinical progress}

Repeat 24-hour urinary cortisol level, a month later, was 779 nmol (NR 138-690) and ALT was 86 U/L. Ketoconazole dose was increased to maximum tolerable dose of 1,200 mg daily.

In June, the patient developed persistent cough, shortness of breath and fever. RT-PCR came back positive confirming SARSCoV-2 infection. He was admitted to the local hospital and managed as per national guidelines. He was categorised to have severe COVID-19 pneumonia. Key results are shown in Table 1. He was treated with supplementary oxygen, broad spectrum antibiotics, methylprednisolone, prophylactic doses of enoxaparin and regular proning. He developed severe hyperglycaemia requiring high dose intravenous insulin infusions before switching to twice daily insulin regimen. Following discharge, he endured a protracted period of convalescence with fluctuating blood pressure, poorly controlled diabetes, severe fatigue and increased soft tissue swelling.

During endocrine review in September, he was noted to have further weight gain now reaching $96 \mathrm{~kg}$ (BMI $32.8 \mathrm{~kg} / \mathrm{m}^{2}$ ), worsening abdominal adiposity and widespread limb bruising. He had also developed bilateral pedal oedema up to the shin. Repeat 24-hour urinary cortisol level was persistently high at 1,190 nmol with ALT $70 \mathrm{U} / \mathrm{L}$.

He underwent desmopressin-stimulated IPSS which failed to demonstrate central to peripheral ACTH gradient, thus suggesting an ectopic source of ACTH secretion. Contrast-enhanced computed tomography (CT) of the chest, abdomen and pelvis did not show any obvious pathology apart from bulky adrenal glands. Due to local unavailability of nuclear scintigraphy, he was

\begin{tabular}{lcc} 
Table 1. Key test results at time of admission with \\
SARS-CoV-2 infection & & \\
\hline Test & Results & Reference range \\
Interleukin 6, pg/mL & 12.21 & $<7$ \\
C-reactive protein, $\mathrm{mg} / \mathrm{dL}$ & 96 & $<10$ \\
White cell count, $\times 10^{9} / \mathrm{L}$ & 15.6 & $4.0-11.0$ \\
Lymphocytes, \% & 17 & $20-40$ \\
Erythrocyte sedimentation rate, & 26 & $1-13$ \\
mm/Hr & & \\
Ferritin, $\mu \mathrm{g} / \mathrm{L}$ & 382.7 & $10-150$ \\
Lactate dehydrogenase, U/L & 405 & $135-225$ \\
D-dimer, $\mu \mathrm{g} / \mathrm{mL}$ & 0.3 & $<0.4$ \\
Prothrombin time, seconds & 12 & $11-13.5$ \\
Urea, $\mathrm{mg} / \mathrm{dL}$ & 15 & $5-20$ \\
Creatinine, mg/dL & 1.01 & $0.84-1.21$ \\
Alanine aminotransferase, U/L & 86 & $7-56$
\end{tabular}


sent to another facility for Ga-68 DOTANOC positron emission tomography / CT. It also failed to reveal tracer avid lesion to account for ACTH secretion.

Clinically, the patient was in florid Cushing's syndrome with severe fatigue, rapid weight gain, worsening soft tissue oedema, erratic glucose control and muscle wasting. He was very depressed and also reported erectile dysfunction and low libido since initiation of ketoconazole.

\section{Definitive treatment and outcome}

Due to florid Cushing's syndrome with high risk of mortality, inadequate response to medical therapy and with no operable ectopic ACTH source, it was decided to proceed with bilateral adrenalectomy to rapidly ameliorate hypercortisolism. He underwent an uneventful laparoscopic procedure under perioperative steroid cover. He was discharged 3 days later with reducing schedule of prednisolone to maintenance daily dose of $5 \mathrm{mg}$ with fludrocortisone of $100 \mu \mathrm{g}$.

At the 3-week postoperative review, there was a significant reduction in facial oedema with complete resolution of pedal oedema. Weight loss of $5 \mathrm{~kg}$ was noted compared with preoperative measurement. Fasting glucose was $5.7 \mathrm{mmol} / \mathrm{L}$ off anti-diabetic medications. Similarly, blood pressure was $130 / 80 \mathrm{mmHg}$ off all antihypertensives. He reported improvement in muscle aches and energy level and had started to walk short distances unaided. Mentally he felt much better and had hoped to come off anti-depressants in the near future. He received education on sick day rules and advised to carry medic alert card. He will be followed up with interval imaging to identify the occult neuroendocrine tumour.

\section{Discussion}

Ectopic ACTH secretion (EAS) by various solid tumours of neuroendocrine origin makes up $10 \%$ to $20 \%$ of cases of ACTH dependent hypercortisolism. ${ }^{3}$ Over half of them are found in the lung and thymus with the most common being bronchial carcinoid tumours (over $25 \%$ ) followed by small cell carcinoma (20\%). Other sites of origin include medullary thyroid cancer, pheochromocytoma and islet cell tumours. Rarely extra thoracic non endocrine tumours may be the culprit. ${ }^{4}$ Differentiating EAS from pituitary source and localising lesion is crucial to guide definitive surgical treatment, since resection of adenoma or ectopic tumour has a high likelihood of clinical and biochemical cure. IPSS remains the most accurate test to differentiate between pituitary versus ectopic source of ACTH secretion. ${ }^{3,5}$ Localisation of ectopic tumour is another challenge necessitating application of various imaging modalities. Despite thorough radiological evaluation, substantial proportions of ectopic tumours remain unlocalised. ${ }^{6,7}$ In such cases, use of adrenolytic medications such as ketoconazole and metyrapone to control hypercortisolaemia and eventual adrenalectomy becomes main therapeutic strategy since adrenolytics are associated with significant side effects including serious liver dysfunction. ${ }^{8}$ Moreover, many ectopic tumours are indolent and may take as many as 20 years to be identified. ${ }^{4}$

There are some unique features to our case. It came at a time of global pandemic disrupting routine clinical services. While the European Society for Endocrinology (ESE) produced recommendations on the management of Cushing's syndrome during the pandemic with a focus on providing medical control of hypercortisolism and deferring invasive procedures except in cases with high suspicion of EAS, there is no literature to guide management of SARS-CoV-2 infection with active Cushing's syndrome. ${ }^{9}$ Indeed, to the best of our knowledge, ours is the first case report of hypercortisolism secondary to EAS contracting SARS-CoV-2 infection.

Hypercortisolism is chronic inflammatory state with impaired innate and adaptive immunity predisposing patients to viral and other infections. While chronic hypercortisolism is associated with increased cytokine secretion which may be a risk for cytokine release syndrome and ARDS, the robust inflammatory response to acute infections is not always observed..$^{10,11,12}$ One would speculate therefore that patients with Cushing's syndrome may have lower risk of acute respiratory distress syndrome development with SARS-CoV-2 infection, however, there is paucity of good quality data to confirm this. Moreover, cortisol excess associated comorbidities of obesity, diabetes and hypertension are recognised risk factors for adverse outcome in COVID-19. ${ }^{12,13}$ Additionally, data is rapidly accumulating in regards to severe insulin resistance, reduced insulin secretary capacity and metabolic decompensation with COVID-19. ${ }^{14,15}$

The combination of Cushing's syndrome and concurrent SARSCoV-2 infection is, therefore, a perfect storm requiring heightened vigilance in caring for this cohort of patients with meticulous attention to foresee and manage complications.

Further large-scale studies are required to add to the evidence and provide further clarity in our understanding of COVID-19 management and clinical outcomes in patients with active Cushing's syndrome.

\section{Acknowledgements}

The author would like to record his admiration for the courage displayed by the patient throughout his illness. The patient strongly supports sharing his case for the benefit of medical community and patient care. The author would like to thank his colleagues, Dr Osama Ishtiaq and Dr Umar Yousaf Raja, for their valuable input during clinical management and Dr Hadi Khan who performed the adrenalectomy.

\section{References}

1 Ragnarsson O, Olsson DS, Chantzichristos D et al. The incidence of Cushing's disease: a nationwide Swedish study. Pituitary 2019;22:179-86.

2 Arnaldi G, Angeli A, Atkinson AB et al. Diagnosis and complications of Cushing's syndrome: a consensus statement. J Clin Endocrinol Metab 2003;88:5593-602.

3 Isidori AM, Kaltsas GA, Pozza C et al. The ectopic adrenocorticotropin syndrome: clinical features, diagnosis, managment and long-term follow-up. J Clin Endocrinol Metab 2006;91:371-7.

4 Isidori AM, Lenzi A. Ectopic ACTH Syndrome. Arq Bras Endocrinol Metabol 2007;51:1217-25.

5 Kaltsas GA, Giannulis MG, Newell-Price JD et al. A critical analysis of the value of simultaneous inferior petrosal sinus sampling in Cushing's disease and the occult ectopic adrenocorticotropin syndrome. J Clin Endocrinol Metab 1999;84:487-92.

6 Doppman JL, Nieman L, Miller DL et al. Ectopic adrenocorticotropic hormone syndrome: localization studies in 28 patients. Radiology 1989;172:115-24.

7 Torpy DJ, Chen CC, Mullen N et al. Lack of utility of (111) In-pentetreotide scintigraphy in localizing ectopic ACTH producing tumors: follow-up of 18 patients. J Clin Endocrinol Metab 1999:84:1186-92. 
8 Nieman L, Biller B, Findling J et al. Treatment of Cushing's syndrome: an Endocrine Society clinical practice guideline, J Clin Endocrinol Metabol 2015;100:2807-31.

9 Newell-Price J, Nieman L, Reincke M, Tabarin A. Endocrinology in the time of COVID-19: management of Cushing's syndrome. Eur ] Endocrinol 2020;183:G1-7.

10 Hasenmajer V, Sbardella E, Sciarra F et al. The Immune System in Cushing's Syndrome. Trends Endocrinol Metab 2020;31:655-69.

11 Pivonello R, Isidori AM, De Martino MC et al. Complications of Cushing's syndrome: state of the art. Lancet Diabetes Endocrinol 2016:4:611-29.

12 Guarnotta V, Ferrigno R, Martino M et al. Glucocorticoid excess and COVID-19 disease. Rev Endocr Metab Disord 2020 [Epub ahead of print].

13 Zhang JJY, Lee KS, Ang LW et al. Risk factors for severe disease and efficacy of treatment in patients infected with covid-19: a system- atic review, meta-analysis, and meta-regression analysis. Clin Infect Dis 2020;71:2199-206.

14 Pal R, Bhadada SK. COVID-19 and diabetes mellitus: An unholy interaction of two pandemics. Diabetes Metab Syndr 2020;14:513-7.

15 Azar WS, Njeim R, Fares AH et al. COVID-19 and diabetes mellitus: how one pandemic worsens the other. Rev Endocr Metab Disord 2020;21:451-63.

Address for correspondence: Dr Tejhmal Rehman, Department of Endocrinology and Diabetes, Shifa International Hospital, 4 Pitrus Bukhari Road, H-8/4 Islamabad, Pakistan.

Email: tejhmal@yahoo.com

Twitter: @tejhmal 